\title{
PCA and LDA Method with Neural Network for Primary Diagnosis of Genetic Syndrome
}

\author{
Dhiren Pandit ${ }^{1}$, Dr. Jayesh Dhodiya ${ }^{2}$ \\ Assistant Professor, Science \& Humanities Department, LDRP-ITR, Gandhinagar, India ${ }^{1}$ \\ Assistant Professor, Applied Mathematics and Humanities Department, SVNIT, Surat, India ${ }^{2}$
}

\begin{abstract}
Dysmorphic diseases are the result of the congenital malformation and abnormal facial features. These abnormal facial features were used to diagnose the disease before cyto or molecular techniques are explored. A high degree of experience and expertise are essential for the identification of such diseases that includes Cleft palate, anencephaly and agenesis of limb etc. The correct genotype-phenotype correlation is a labour intensive especially for rare diseases. As the face development is controlled by several genes, it provides important hints for initial identification of genetic anomalies. The use of a computer based pre-diagnosis system can offer effective decision support for the less explored field like dismorphic diseases. Certain mathematical algorithms are there which can be used to analyse the facial features that diagnose the syndrome accurately. In this work we develop and demonstrate that accurate classification of dysmorphic faces is feasible by image processing of two dimensional face images. We test the proposed system on patient image data by constructing a dataset of dysmorphic faces published in scholarly journals, hence having accurate diagnostic information about the syndrome. Our methodology represents facial image data in terms of principal component analysis (PCA) and Linear Discriminant analysis (LDA) and classification is done using Neural network. This method has been tested with 03 syndromes with few images per syndrome. A diagnosis success rate of $87 \%$ has been established. It can be concluded that a great number of syndromes indicating a characteristic pattern of facial anomalies can be typically diagnosed by employing computer-assisted machine learning algorithms.
\end{abstract}

Keywords: Principle component analysis (PCA), Linear Discriminant analysis(LDA); Neural network(NN).

\section{INTRODUCTION}

Dysmorphology is the study of human congenital defects and abnormalities of body structure that originate before birth. In Dysmorphology the "Dys" means disordered or abnormal and "Morph" means shape. The patients having this syndrome can be identified through the combination of congenital malformations which is a primary defect associated with the basic alteration in the structure and unusual facial features [1]. To identify such patients necessitate a high degree of experience and expertise as dysmorphic diseases are unusual [2] e.g. cleft palate, anencephaly and agenesis of limb. The human brain has the capacity to recognize what is familiar or unfamiliar in a face and hence people can identify the individual shape of the patients with Downs syndrome. The expert clinical geneticists develop this natural skill at such a degree that they distinguish the delicate facial features associated with several hundred dysmorphic syndromes [3]. The Dysmorphologic diagnosis is usually performed by general practitioners, dermatologists or pediatricians due to the scarcity of the trained geneticists. The diagnosis is performed by referring the images or terms standardized and specified in some limited number of catalogues and databases. Due to the limited knowledge of these diseases, in the rural areas there is a delay in the diagnosis of these rare but deadly diseases. This can lead to diagnostic inaccuracy that limit the right cure of the patients and the correct. guiding of their parents who may be at risk of having a new dysmorphic child. The process reaching to the right diagnosis can be very lengthy and struggling which often gives the experience of the burdensome anxiety and frustration.
Moreover delay in this diagnostic process may also delay in treatment and hence the diagnosis is of clinical importance for several reasons according to Smithson [1]. First the knowledge of the anomalies associated with particular syndrome direct towards the investigations to be undertaken that improves the patient management; second, the proper diagnosis leads to the proper treatment and the good prognosis. E.g. bone marrow transplantation is given to cure some inborn errors of metabolism. Third, it determines the appropriate guidance regarding to the genetic risks and possible prenatal diagnosis. Thus delay in early diagnosis and treatment, have a sad impact on patients health. Therefore, thorough genetic diagnosis at an early stage is crucial that will ensure an overall improvement in the patient's quality of life and care. The face develops under the influence of many genes that distinguishes a person at first glance. Facial features give lots of clues about the identity, gender, age and ethnicity. Hence the face provides the important hints for initial identification of genetic anomalies and dysmorphologic syndrome. Certain properties of facial dysmorphology are used to pre-diagnose the syndrome before clinical examination. It might be possible to diagnose a good number of syndromes correctly by using computer assisted face analysis DSS as asserted by some scientists such as Farkas [4], Loos [5], Boehringer [2], and Hammond [6, 7]. Farkas [4] pioneered techniques for studying facial morphology using direct anthropometry for nearly 40 years ago [4]. Douglas [8] has stated that classification of faces based on facial patterns in isolation is unlikely to be accepted by dysmorphologists unless the mathematical 
features extracted and identified by feature selection algorithms to be discriminatory can be related to facial appearance. Principal component analysis (PCA), independent component analysis (ICA), kernel principal component analysis (KPCA), local feature analysis (LFA), probability density estimation (PDA), multi-linear analysis (MLA), elastic graph matching (EGM), kernel discriminant analysis (KDA), support vector machine (SVM), Gabor wavelet and Fischer's linear discriminant analysis (LDA) exist to analyze the features of a face. Among them, the methods of PCA using eigenface, elastic graph matching, Gabor wavelet and Fischer's LDA are popular for face recognition. PCA can be computed as an optimal compression scheme that minimizes the mean squared error between an image and its reconstruction [9] as well as it may achieve good results of up to 96 percent recognition under ideal conditions [10] which can be provided with either capturing images in ideal environments by having a good illumination and using a good capturing device or pre-processing of images with several image processing techniques before extracting features.

\section{PCA+LDA FUSION METHOD WITH NEURAL NETWORK}

PCA+LDA fusion method with Neural network shown in Figure 1 contains five different levels of recognition process which are as follows.

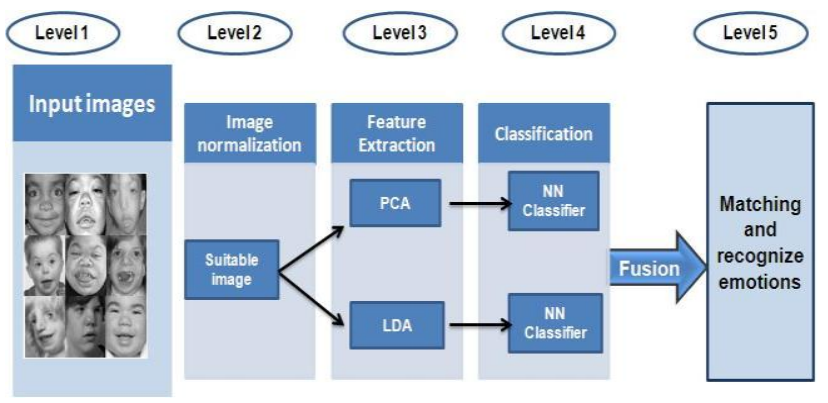

Fig 1 Recognition process

At the initial stage multiple face images of different persons with different syndromes are entered in proposed system. These sets are normalized at Level 2 by applying different normalization method like, Gamma Correction [11], RGB to Gray Scale conversion, enhancement of contrast and Resizing Image [11]. At Level 3 suitable Eigen faces are extracted parallel by the methods Principle Component Analysis (PCA)[12] and Linear Discriminant Analysis (LDA). At Level 4 the eigen faces of both PCA and LDA are classified by Neural Network algorithm with Multilayer feed forward and Back propagation algorithm of artificial neural network. At this level the images are classified according to the different syndromes and it is called as training set.

Here the newly entered image is classified on the basis of this training set for respective syndrome. At Level 5 the output (Classified Images of syndrome) by neural network are combined and system matches the final output with stored image sets and will exposed the suitable syndrome.

\section{A. Face detection (Level -1)}

This is Level 1 of proposed system in which face images of different persons with syndromes are given as input images. Frontal face images belonging to syndromes can be captured from images stored in a folder and hardcopy pictures as well as from real-time images which can be detected simultaneously from patients via a camera attached to the computer. The images can be automatically captured on an ordinary high resolution digital camera (e.g. 8 megapixels) mounted across the patient (no matter what the distance is), provided that a frontal face is detected. Thus, preset frontal faces are ready to be treated requiring no pre-processing or data preparation. Frontal faces are the essential components of our data preparation and model building.

\section{B. Image Normalization (Level -2)}

This elaborates the techniques to normalize the images in order to increase the efficiency of feature extraction and classification of syndromes. To normalize the image following set of mathematical techniques are adopted.

1) Gamma correction

2) Dimension reduction - RGB to gray

3) Histogram equalization

4) Image Resizing

1) Gamma Correction (on RGB Image): Gamma correction balance darkness and lightness of an image, which improve the visibility of an image. Especially in facial feature extraction it is necessary that the prime feature of face area must be visible separately from the other part of the image. In MATLAB gamma value varies between 0.0 and 10.0 as gamma value moves towards 0.0 the lightness will be increased, it moves towards 10.0 the darkness will be increased and while gamma value is 1.0 there will no changes in image.

Gamma function: If $X$ is an original value of an image then the new value of $\mathrm{X}$ is obtained as;

$$
\mathrm{X}_{\mathrm{NEW}}=\mathrm{X}^{\mathrm{Gamma}}
$$

2) Dimension Reduction: (RGB to Gray): Basically the coloured image has three dimensional array pixel value $\mathrm{M}$ * $\mathrm{N} * 3$ of Red, Green and Blue (RGB)[11]. It is quite difficult to perform image processing technique on three dimensional matrix in MATLAB, so for convenient and smoother processing the image is converted in gray format. A gray image has two dimensional $\mathrm{M}^{*} \mathrm{~N}$ pixel value which lies between $[0,255]$ as per the gray value of an image pixel. $I=\operatorname{rgb} 2 \operatorname{gray}(\mathrm{RGB})$ converts the true colour image RGB (assumed to be in the RGB colour space) to the grayscale image I. The theory behind the rgb2gray MATLAB function is to convert a true colour image from the RGB colour space to the YIQ colour space, and then take the value of the $\mathrm{Y}$ component as the grayscale value. The argument is that in the YIQ colour space the luminance $(\mathrm{Y})$ represents the grayscale information while hue (I) and saturation $(\mathrm{Q})$ represents the color information. The MATLAB function to perform this operation is:

$$
\text { IamgeGray = rgb2gray (ImageGamma); }
$$


3) Histogram Equalization: In feature extraction it is essential that the gray image must have more dissimilarity so that the gray values of each pixel become considerably diverge from each other. The better variation in range $[0$, 255] makes the identification feature better. The Histogram Equalization [11] enhances the contrast of images by transforming the values in an intensity image so that the histogram of the output image is approximately flat. The histogram of a digital image with $\mathrm{L}$ total possible intensity levels in the range $[0, \mathrm{G}]$ is defined as the discrete function

$$
\mathrm{h}_{\mathrm{r}_{\mathrm{k}}}=\mathrm{n}_{\mathrm{k}}
$$

where, $r_{k}$ is the $\mathrm{k}^{\text {th }}$ intensity level in the interval $[0,255]$ and $n_{k}$ is the number of pixels in the image whose intensity level is $r_{k}$. The value $G$ is 255 for image of class uint8, 65535 for images of class uint16, and 1.0 for image of class double. Here $r_{1}$ corresponds to intensity level $0, r_{2}$ corresponding to intensity level 1 , and so on, with $r_{L}$ corresponding to level $\mathrm{G}$. Note also $\mathrm{G}=\mathrm{L}-1$ for images of class uint 8 and uint16. Often it is useful to work with normalized histograms, obtained simply by dividing elements of $h_{r_{k}}$ by the total of pixel in the image, which we denote by $n$.

$$
\mathrm{p}\left(\mathrm{r}_{\mathrm{k}}\right)=\frac{\mathrm{h}\left(\mathrm{r}_{\mathrm{k}}\right)}{\mathrm{n}}=\frac{\mathrm{n}_{\mathrm{k}}}{\mathrm{n}}
$$

We assume that the intensity levels are continuous quantities Normalized to the range [0,1], and $\mathrm{p}_{\mathrm{r}}(\mathrm{r})$ denote the probability density function of the intensity level in a given image, where the subscript is used for differentiating between the PDFs of input and output images. If we perform the transformation on the input level to obtain output intensity levels s.

$$
\mathrm{s}=\mathrm{T}(\mathrm{r})=\int_{0}^{\mathrm{r}} \mathrm{p}_{\mathrm{r}}(\mathrm{w}) \mathrm{dw}
$$

Where, $w$ is a dummy variable of integration. It can be shown that the probability density function of the output levels is uniform. The MATLAB function to perform this operation is Image-

Histogram $=$ histeq (ImageGray, hgram);

4) Image Resizing: Since different face images are considered, there is a possibility that the different face images are extracted in different sizes, and also for image processing in MATLAB the dimensions of each image are required to be same. To make the image process smoothly it is necessary to make all the syndrome images same sized, as per desired but not so small not so big and in sufficient sized that never affect the quality of result [11].The MATLAB function to perform this operation is. Resize=imresize(ImageHistogram,[numrows,numcols])

\section{Feature Extraction (Level -3)}

Different mathematical techniques are available for feature extraction, here we have used (I) Principle Component Analysis and (II) Linear discriminant Analysis approaches on same images with syndromes.

1) PCA method for Syndrome Recognition: Let $X$ be a d-dimensional feature vector. Here $d$ is equal to the number of pixel of each face image. Therefore methods for reducing the dimensionality of such image space are required. Principal Component Analysis (PCA) and Linear Discriminant Analysis (LDA) are used for dimensionality reduction. Principal Component Analysis [13] [14] is defined by the transformation:

$$
\mathrm{y}_{\mathrm{i}}=\mathrm{W}^{\mathrm{T}} \mathrm{x}_{\mathrm{i}}
$$

$x_{i} \in X \subseteq R^{d}, \mathrm{i}=1,2,3, \ldots, \mathrm{n}$ (n samples). W is a ddimensional transformation matrix whose columns are the eigenvectors related to the eigenvalues computed according to the formula:

$$
\lambda e_{i}=S e_{i}
$$

$\mathrm{S}$ is covariance matrix defined by

$S=\sum_{i=1}^{n}\left(x_{i}-m\right)\left(x_{i}-m\right)^{T}, m=\frac{1}{n} \sum_{i=1}^{n} x_{i}$

This transformation is called Karuhnen-Loeve transform. It defines the d-dimensional space in which the covariance among the components is zero. Hence it is possible to consider a less number of "principal" components exhibiting the highest variance. In the face space, the eigenvectors related to the most expressive features are called "eigen faces"

2) LDA methods for Syndrome Recognition: The Linear Discriminant Analysis is defined by the transformation:

$$
y_{i}=W^{T} x_{i}
$$

The columns of $\mathrm{W}$ are the eigenvectors of $S_{W}^{-1} S_{b}$, where $S_{W}$ is the within-class scatter matrix, and $S_{b}$ is the between-class scatter matrix. It is possible to show that this choice maximizes the ratio $\frac{\operatorname{det}\left(S_{b}\right)}{\operatorname{det}\left(S_{W}\right)}$. These matrices are computed as follows:

$S_{\mathrm{W}}=\sum_{j=1}^{c} \sum_{i=1}^{n_{j}}\left(x_{i}^{j}-\mu_{j}\right)\left(x_{i}^{j}-\mu_{j}\right)^{T} ; \mu_{j}=\frac{1}{n_{j}} \sum_{i=1}^{n_{j}} x_{i}^{j}(9)$

Where $x_{i}^{j}$ is $i^{t h}$ pattern of $j^{\text {th }}$ class and $n_{j}$ is the number of patterns for the $j^{\text {th }}$ class. The eigenvectors of LDA are called "fisherfaces". LDA transformation is strongly dependent on the number of classes (c), the number of samples (n), and the original space dimensionality (d) . It can be shown that there are almost $c-1$ nonzero eigenvectors. $c-1$ being the upper bound of the discriminant space dimensionality. We need $d+c$ samples at least to have a nonsingular $S_{W}$. It is impossible to guarantee this condition in many real applications. Consequently, an intermediate transformation is applied to reduce the dimensionality of the image space. For this we used the PCA transform.

\section{Classification (Level -4)}

At Level 3 eigen features of normalized images have been obtained by PCA and LDA which are classified for syndrome recognition at this level using neural network (NN) individually. At Level 4 neural network is trained to classify PCA and LDA eigen images by using Multilayer Perceptron (MLP) with Back Propagation classification algorithm. In this level the NN parameters like, Error surface, number of hidden layers, learning rate, momentum, input standardization, weight initialization, 
training and stopping criteria, generalization of $\mathrm{NN}$ is decided.

\section{E. Fusion of PCA-NN and LDA-NN (Level -5)}

After Level 4 the PCA-NN and LDA-NN classified syndrome images are fused in this Level 5 using score based strategy discussed as under. If the score functions are directly comparable or if there exists at least an acceptable transformation scheme to make the involved classifiers comparable, score based strategies are good ways for decision process. In this work, NN is used as a classifier for both systems (PCA and LDA), hence outputs of both systems are in same format, so we select score based strategy (SBS) as combiner

\section{Algorithm of Score Based Strategy (SBS) [15]}

Step 1 Assemble Label, PCA-NN and LDA-NN Score(S) of syndrome

Step 2 Set threshold values (TV) for PCA-NN and LDANN

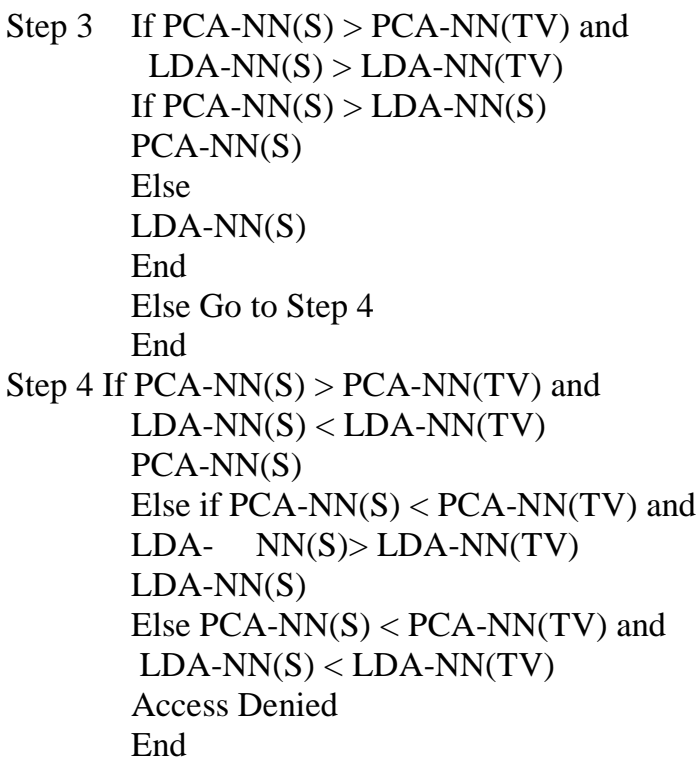

Where, TV = Threshold Value, $\mathrm{S}=$ Score $]$

Neural Network gives the individual PCA and LDA accuracy score of classification of a syndrome in percentage, which are used as PCA-NN and LDA-NN scores at Step 1 of algorithm. The threshold value is consider as expected accuracy we want from the NN classification as per our expectation that can be set manually, for both PCA-NN and LDA-NN classifier at Step 2 which can be same or differ. In Step 3 conditional approach is used for both PCA-NN and LDA-NN, if both classification scores crossed their limit of threshold value then the higher classified score is accepted otherwise in failure situation it indicates not to reach the expected level of accuracy. In winning situation if one of the classifier crosses the threshold limit at Step 4 then its accuracy score will be considered as final matching score.

Recognition (Level-5) After fusion of PCA-NN and LDA-NN, to identify a face, system needs the training set of face images of persons. At this level we consider face images according to the class of different persons (E1,E2, ...,Ek) by NN with same feature extraction techniques (PCA and LDA) is called as "Training Set" which have been already stored in our database,. The more customized face images makes the database stronger for highly accurate classification, so the update in training set is much essential for syndrome recognition. When the new face image of a person with syndrome or sequence of images are entered as test image, at that time proposed method performs all processes of Levels 1 to 4 on it and obtained an output which is matched with all the stored face class(E1,E2, ...,Ek) of an individual. At this level system finds the association between output face and stored classes of categorized faces then identifies the correct syndrome.

\section{DATABASE AND DESIGN OF MODEL}

The study sample was composed of 3 syndromes comprising 62 frontal faces that are depicted in Figure 3. Our set of patients includes both sexes and their age range is from newborn to 5 years old, and some images are suboptimal with regard to pose, mimic, and lighting. These syndromes were trained by the all database explained previously. Generated eigenfaces are displayed in Figure 3All paragraphs must be indented. All paragraphs must be justified, i.e. both left-justified and right-justified.

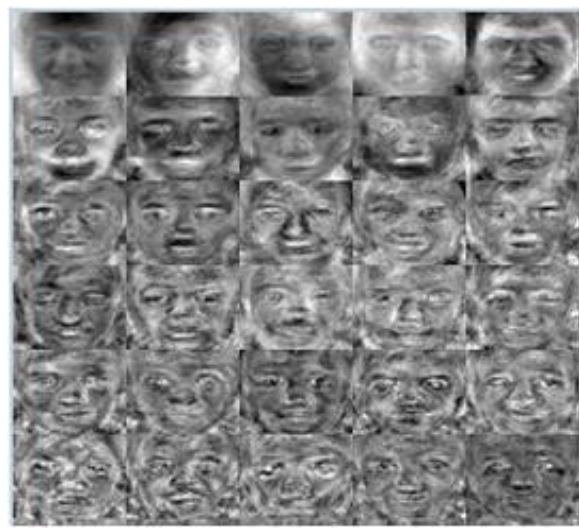

Figure 2 Eigen Faces

The test images were put into a folder and each test image as compared to other images in the database to measure how close it is on the vector space to others.

\section{RESULTS AND DISCUSSION}

Here we have considered the face images of different persons for recognition of syndrome, which we call as train images. From these images our algorithm is trained for three different classes of syndromes. Then we normalize sequence of images. Here we did classification using Neural network.

\section{A. Flow of Process}

Following is the flow of entire process of Classification of person with syndrome. Entire process is divided in five different levels.

\section{Level 1 Input Image sequence}

At first level sequence of test face images with syndromes are given as input. Here in train images we have consider all face images with three different syndromes and test images for which we are seeking recognition of person. 


\section{$>\quad$ Level 2 Image Normalization}

In second level normalization of face image is done. Here face image is crop according to face portion of a person in that image. After these images are converted to grey scale images for ease of calculation.

\section{Level 3 Feature Extraction}

We use Principle component analysis and Linear Discriminant analysis for feature extraction. At this level Eigen faces and fisher faces which contains important characteristic of images are extracted from both train and test images.

\section{Level 4 Classification}

At level 4 image classifications is done. For classification purpose we have use Neural network with back prorogation algorithm. We use same classifiers in PCA and LDA both. We call it as PCA+NN and LDA+NN.

\section{$>\quad$ Level 5 Recognition}

In level 5 matching is done by combining both technique $\mathrm{PCA}+\mathrm{NN}$ and $\mathrm{LDA}+\mathrm{NN}$. We have use fusion of PCA and LDA technique for this purpose. And with this classification we obtain recognized syndrome with given face image. And with the help of number of such recognized images we classify number of images.

\section{B. Results}

$>$ For experiment we have taken 62 different images of persons with three different syndromes for train purpose. All these images will train our algorithm for recognition.

$>$ Once algorithm is trained we have taken test images for three syndromes.

$>$ Figure 3 are few images extracted from set of train Images

$>$ We have passed 100 test images of different persons with syndrome as input and obtained the following results.

> Out of 100 images there are 87 images are correctly classified by PCA+LDA fusion method using neural network.

$>$ Hence we get 87 percentage classification rate for this set of train and test images.

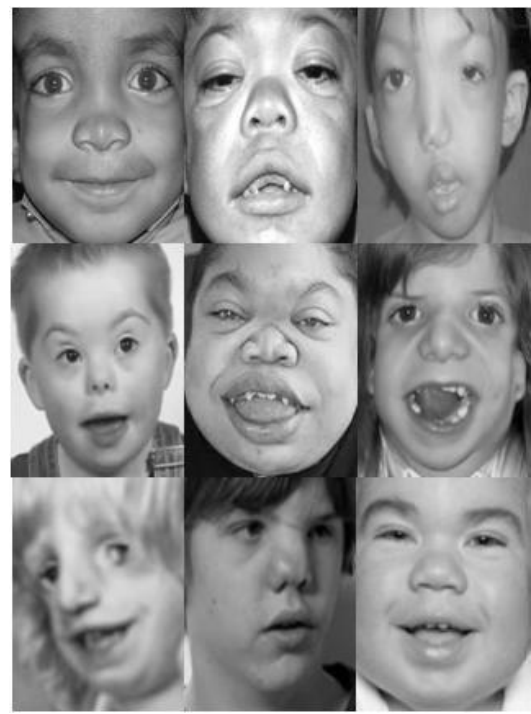

FIGURE3. TRAIN IMAGES

\section{Conclusion}

We used PCA+LDA method with classification using neural network to recognize person with genetic syndrome.

> $\mathrm{PCA}+\mathrm{LDA}$ method with classification using neural network gives very good recognition rate.

This method is used to analyze the facial features that diagnose the syndrome accurately

Hence PCA+LDA fusion method with classification using neural network is good tool for primary recognition of person with genetic syndrome.

\section{REFERENCES}

[1] Smithson, S. F., and Winter, R. M., "Diagnosis in Dysmorphology: Clues from the Skin" The British Journal of Dermatology, 151(5), 953-60, 2004

[2] Boehringer S., Vollmar T., Tasse C. and Wurtz R. "Syndrome identification based on 2D analysis software." European Journal of Human Genetics, vol. 14(10), 1082-1089, 2006

[3] Hammond P. "What's in a face?" http://www.hospitalmanagement.net/features/feature1767/, 2011

[4] Farkas, L. G., "Anthropometry of the head and face" New York, Raven Press, 1998

[5] Loos, H. S., Wieczorek, D. and Wurtz R. P. "Computer-based recognition of dysmorphic faces" Eur J Hum Genet, 11(8), 555-60, 2003

[6] Hammond, P., Hutton, T. J. and Allanson, J. E. "Discriminating power of localized three-dimensional facial morphology." Am J Hum Genet, 77(6), 999-1010, 2005

[7] Hammond P. "The use of 3D face shape modelling in dysmorphology." Arch Dis Child, 92(12), 1120-1126, 2007.

[8] Douglas, T. S. and Mutsvangwa, T. E. M., "A review of facial image analysis for delineation of the facial phenotype associated with fetal alcohol syndrome." Am J Med Genet Part A, 152A(2), 528-536, 2010

[9] Kapoor S., Khanna S., and Bahatia R., "Facial gesture recognition using correlation and mahalanobis distance." International journal of computer science and informationsecurity, 7(2), 267-272, 2010

[10] Turk M. A., and Pentland A. P. "Face recognition using eigenfaces.", IEEE Conf.on Computer Vision and Pattern Recognition, 586-591, 1991

11] Gonzalez R., "Digital Image Processing Using MATLAB" Publisher-Tata McGraw-Hill Education, ISBN 0070702624 9780070702622, 2013

[12] Tunglin D. "Facial Expression Classification Using PCA and Hierarchical Radial Basis Function Network" Journal of Information Science And Engineering, 2006

[13] Turk M. and Pentland A. "Eigenfaces for Recognition" Journal of Cognitive Neuroscience, Vol.3, 71-86, 199

[14] Duda R.O., Hart P.E. and Stork D.G. "Pattern Classification" John Wiley Sons, USA, 2001

[15] Pandit D., Dhodiya J., "PCA+LDA Method for Face Recognition Using Neural Network" International Journal of Innovative Science and Modern Engineering, Vol.3, Issue-6, 6-11, 2015 